\title{
Types of Anatomic Conformity of Cardiac Conduction System
}

\author{
Spirina Galina Alekseevna \\ Department of Human Anatomy, Ural State Medical University, Street Repina 3 Sverdlovsk Region, Yekaterinburg 620000, Russian \\ Federation
}

\begin{abstract}
Anatomical features of portions of the conduction system in connection with parameters and shape of the heart, ventricles and parts of the IVS (interventricular septum) were studied on 501 specimens oh human hearts of both sexes and different ages using common morphological methods. In prenatal and postnatal periods of ontogenesis are similar variants of the structure of the ventricles of the heart with certain proportions of the linear dimensions inflow and outflow sections, parameters parts of IVS. Each of options of structural organization of heart ventricles and its IVS corresponds to set of specific features of portions of cardiac conduction system, signifying change of the positional angles, linear dimensions and shape.
\end{abstract}

Key words: Heart, structure, conduction system.

\section{Introduction}

Development of surgical treatment methods of arrhythmia and congenital heart defects to a large extent by the correct assessment of the CCS (cardiac conduction system) structural variability [1-3]. Only a few studies provide data on the principles of systematization of the conduction system of the heart [4]. The aim of this work is to study individual differences in position, size and shape of parts of the CCS atrioventricular portion in terms of age and with regard to the cardiac structure.

\section{Material and Methods}

By applying generally accepted morphological methods in 501 heart specimens of 12-32 weeks gestation fetuses, infants, children and adults of both sexes and different ages, we studied the position, size and configuration of the AV node (atrioventricular node), AV bundle and its branches with regard to the structure of cardiac ventricles and portions of the IVS

Corresponding author: Spirina Galina Alekseevna, doctor of medicine, professor, research fields: morphology of the heart and blood vessels in normal and congenital, heart disease. E-mail: profspirina@yandex.ru. (sinus, trabecular, conica). The identify the atrioventricular part of conduction system of the heart used in combination the following methods: 1) macro-microscopic dissection; 2) methods $\mathrm{N}$. Otsuka, T. Hara [5], N. K. Roberts, D. W. J. Pepin [6]; 3) histological studies with preparation method of serial sections; 4) morphometry was performed interrelated parts of the conduction system and the IVS. Obtained by measuring the absolute numerical values converted into the ratio. The work is based on the principle of simultaneous examination of anatomical properties of the heart, its conduction system (on one and same specimen).

\section{Results}

The following cardiac shapes can be distinguished: long and narrow heart (with index less than 0.8), short and wide heart (with index exceeding 0.95) and heart of intermediate shape (with index equal to 0.8-0.95). The fetuses as during postnatal life have the identical structural variations of cardiac ventricles with certain quantitative correlations of linear dimensions of inflow and outflow portions and properties of the IVS portions. The structural organization of cardiac 
ventricles implements the principle on conformal symmetry as the most common pattern of morphogenesis. Every structural variation of cardiac ventricles corresponds to a set of certain properties of AV node, the AV bundle and its branches, reflecting the changed angles of the position, linear dimensions and shape. Revealed three types of anatomic conformity of the conduction system of the heart. Type I. 1 (heart index 0.8-0.95) characterized by proportional correlation of parameters of IVS portion, its sinus portion having a square shape. AV node is positioned on the central fibrous body, AV bundle is on top of muscular ridge of sinus portion of IVS at angle of $20^{\circ}$ to the horizontal. Left and right branches are on respective sides of IVS. Proximal portions of branches and bundle form angles of approx. $90^{\circ}$, slightly convex upfront (Fig. 1). Type II (heart index 1.1-1.5) is characterized by considerable predominance of width of both heart and sinus septum portion, which is rectangular in shape. Position the $\mathrm{AV}$ node and the bundle is similar to that in type I. Angles at which branches leave AV bundle increase to $110^{\circ}-130^{\circ}$. The configuration of front edge of bundle branches changes, they become concave upfront (Fig.2). Type III anatomic conformity of cardiac conduction system (heart index 0.6-0.79) implies length of heart exceeding its width. Sinus portion of right side of IVS is pentagonal, which is distinguished by disproportional correlation of its parameters and parameters of trabecular and conic portions of IVS.

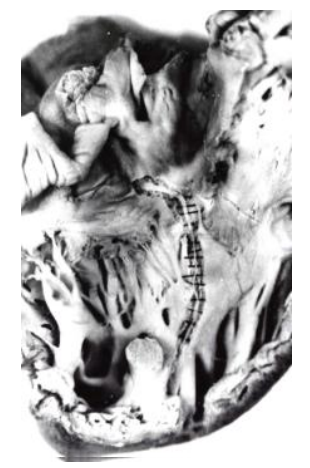

(a) newborn
AV node is positioned at opening of coronary sinus, angle of $\mathrm{AV}$ bundle increases to $45^{\circ}$. Initial parts of branches and bundle form angles from $160^{\circ}-180^{\circ}$, their configuration changes (Fig. 3). Between these types are numerous options relating to the intermediate forms. In all the age groups, regardless of the index of heart prevailed option in which the sine part of the right side of the IVS is rectangular in shape. The sinus portion greater width of its length to 1.2-1.3 times. The length of right ventricular outflow department exceeds the length of the department inflow of 1.2-1.3 times. The left ventricular these departments have the same length. Position AV node, AV bundle is similar to that at I, II types. The initial part of legs form with AV bundle angles of $110^{\circ}-130^{\circ}$, concave to front. Fetus and postnatal ontogenesis revealed the same anatomical variations conformity. It was to the AV bundle and sine of the IVS of the correlation pair. With rectangular shape of the sine part IVS marked variability in the values of the width and length. Form of the sine part, ratio of its parameters can be used as a criterion for estimating the length of preventive $\mathrm{AV}$ bundle without its isolation. Individuals of the same age the smallest length of the bundle detected by the sine part of the square shape, the greatest — with its pentagonal shape.

\section{Discussion}

Observations convinced that anatomy of parts CCS should be studied taking into account the variability of

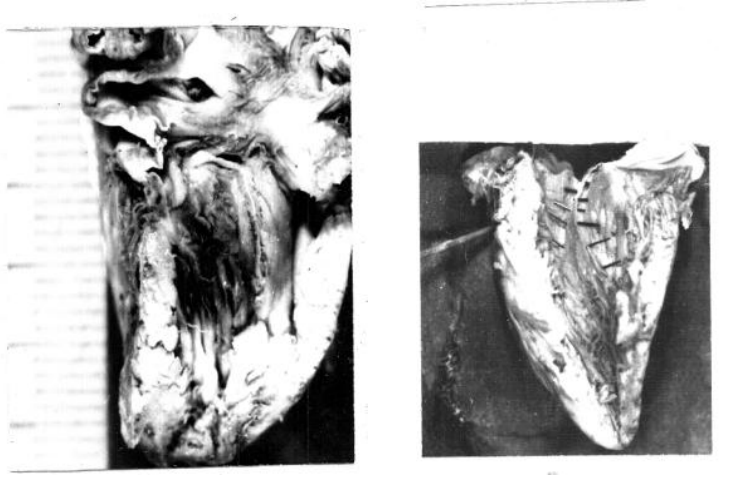

(b) fetus 20 weeks

Fig. 1 Type I of anatomic conformity of cardiac conduction system. 


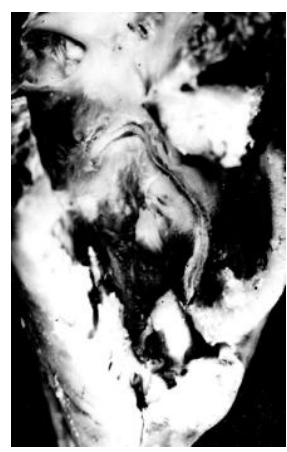

(a) fetus 20 weeks
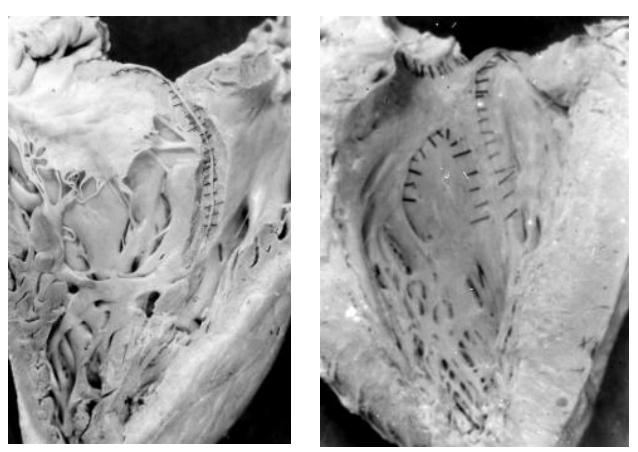

(b) adult

Fig. 2 Type II of anatomic conformity of cardiac conduction system.

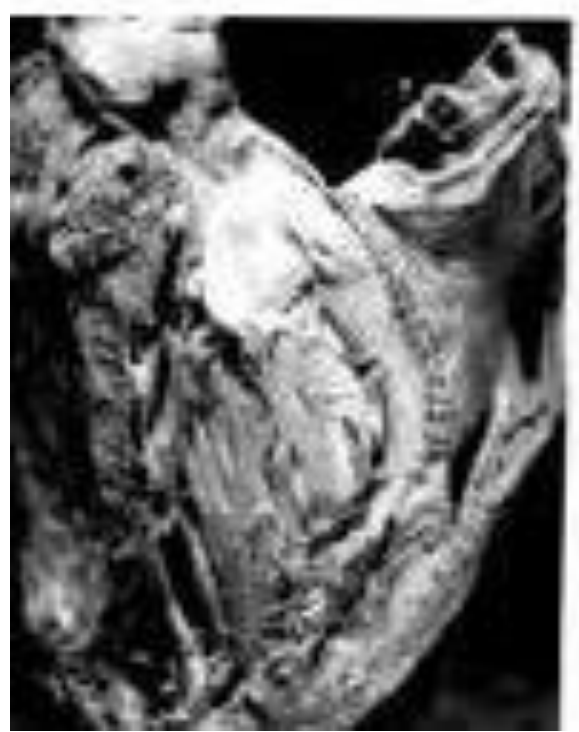

(a) adult

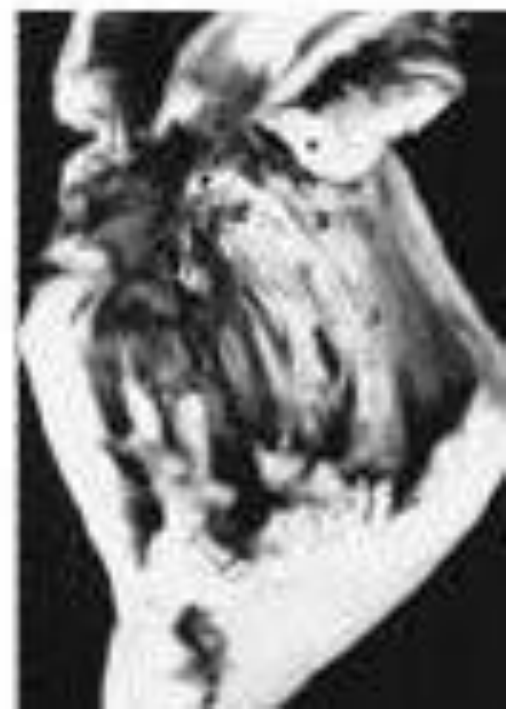

(b) fetus 18 weeks.

Fig. 3 Type III of anatomic conformity of cardiac conduction system.

individual parts of the structure IVS. Analysis of options for the structure of the conduction system should be implemented from the point of ontogenesis. As a result of the work it found that regardless of age, in the pre- and postnatal periods of ontogenesis there are similar options for the structure of the ventricles of the heart to a certain proportion of linear dimensions of parts of inflow and outflow, the parameters of parts of the IVS. The proceedings of the findings confirm G. E. Falkowski, I. I. Berishvili [7] on the independence of the relations between the parameters of parts of the IVS on the size of the heart. Probably the difference in the magnitude of blood flow is due to the variability of proportions ratio between the length departments of inflow and outflow of the ventricles of the heart [8].
The structure of the parts of the IVS, apparently is determined by the genotype of the individual.

\section{Conclusions}

In the prenatal and postnatal periods of ontogenesis are similar variants of structure of the ventricles of the heart with certain proportions of linear dimensions of the sections inflow and outflow, parameters parts of the IVS.

Each of options of structural organization of heart ventricles and IVS corresponds to a set of specific features of portions of cardiac conduction system, signifying change of their positional angles, linear dimensions and shape.

Types of anatomic conformity of cardiac 
conduction system are formed during antenatal life.

The sinus part of the IVS and AV bundle form a correlation pair.

\section{References}

[1] Burakovsky, V. I., Alexi-Meskhishvili, V. V., and Blinova, E. I. 1984. "The Treatment of Children 1 Year of Age with Congenital Heart Disease in a Critical Condition." Cardiology 24 (7): 10-4.

[2] Burakovsky, V. I., and Bokeria, L. A. 1989. Cardiovascular Surgery Guide. Moscow: Medicine. 1-723.

[3] Sharykin, A. C., and Alexi-Meskhishvili, V. V. 1989. "Surgery in Patients under the Age of Three Months." Cardiology 29 (3): 101-3.

[4] Sinev, A. F., and Crimean, L. D. 1985. Surgical Anatomy of the Conduction System of the Heart. Moscow: Medicine, 1-272.

[5] Otsuka, N., and Hara, T. 1965. "Gross Demonstration of the Mammilian Atrioventricular Bundle by a Periodic Acid-Schiff Procedure." Stain. Technol. 40 (3): 305-8.

[6] Roberts, N. K., and Pepin, D. W. J. 1977. "The Atrioventricular Node, His Bundle and Bundle Branches-A New Histologic Techninique." Stain Technolol. 52 (3): 131-5.

[7] Falkowski, G. E., and Berishvili, I. I. 1982. "Morphometric Study of Normal Newborn Heart."Arhive Anatomy 10: 79-85.

[8] Klein, H. W., and Krediet, P. 1984. "Das Herz in Entwicklung, Morphologic und Function.” Anat. Anz. 156: 259-60. 